\title{
Multiple Scattering of Light in a Spherical Cometary Atmosphere with an Axisymmetric Dust Jet
}

\author{
Kenneth M. Chick and Tamas I. Gombosi \\ Space Physics Research Laboralory, University of Michigan. Ann Arbor, Michigan 48109
}

Received February 5, 1992; revised May 15, 1992

We have developed a numerical solution for the anisotropic multiple scattering of light in a cometary atmosphere. The atmosphere is assumed to be a spherical shell illuminated by parallel solar rays. The spatial variation of dust in the coma is symmetric about the Sun-comet axis. Multiple scattering of photons is determined by lambda iteration.

We study two model dust profiles: (1) a spherically symmetric profile and (2) an axisymmetric dust jet at the comet's subsolar point. Calculation is made of the flux of visible energy impinging on the nucleus surface, the mean intensity of visible light throughout the coma, and an approximate solution for the flux of thermal radiation at the comet surface due to emission from dust particles in the coma. We determine conditions under which single and multiple scattering each become significant. Under all conditions examined, at no point on the nucleus surface does the radiant flux exceed the visible flux at the subsolar point of a bare nucleus. $\quad .1992$ Academic Press, Inc.

\section{INTRODUCTION}

Modeling the transfer of visible radiation in a cometary dust coma is an essential element in answering several questions raised by recent observations of Comet Halley. First, analysis of Giotto's Halley Multicolour Camera (HMC) images shows a deviation in the observed scattered light intensity near the nucleus compared to the intensity expected for a dust distribution characteristic of constant radial outflow from a point source (Thomas and Keller 1990, Thomas et al. 1988). This had led to studies concerning the geometric nature of the dust emission from the surface of Halley (Thomas et al. 1988, Huebner et al. 1988, Reitsema et al. 1989a) and to the possibility of dust fragmentation by solar heating (Thomas and Keller 1990). Furthermore, the dust coma appeared quite homogeneous, despite the fact that surface emission seemed concentrated on the sunward side in a few discrete jets, and it has been argued that there should be strong gas-dust flow tangential to the surface of the comet (Keller and Thomas 1989, Keller et al. 1990). Most image interpreta- tion studies to date are based on simple radiative transport models. As part of a general effort to relate coma dust grain dynamics and compositional variation to modeled nucleus emission and dust-gas flow, it would be useful to have a more realistic model of light scattering in a coma, in particular one which would allow for a realistic dust distribution in space, arbitrary viewing geometry, variation of optical properties in the coma, and multiple scattering.

Second, a model of the radiant flux of visible energy to the nucleus surface may help us to understand the process of dust-gas emission from the nucleus. Before Giotto HMC images suggested that the coma dust density was not optically deep, it was proposed that significant negative or positive feedback effects might exist with regard to dust-radiation interaction. In particular, it was thought that light scattering and thermal reemission of energy by coma dust might enhance or reduce the flux of energy to the nucleus surface; this flux, in turn, is the source of heating which causes more dust to be ejected (Hellmich 1981; Weissman and Kieffer 1981; Marconi and Mendis 1984, 1986; Gombosi et al. 1986). In view of the rather shallow optical depths observed at Comet Halley (Keller et al. 1987), the effect of light scattering on the overall radiant flux to the nucleus of Halley is perhaps not a dominant effect, at least at the time of the Giotto observations, but should still be examined. Since dust emission was almost exclusively from the sunward face of Halley, the nucleus is certainly sensitive to insolation, and during violent outbursts scattering effects may play a role. The physical process of dust-gas emission from the nucleus and the reason for the observed sporadic outbursts are poorly understood (Hughes 1991), and it would be useful to quantify the conditions under which scattering effects might play a role.

Light scattering in the coma has been included in many past coma models with numerical techniques that either lack a rigorous foundation and/or are difficult to verify. Hellmich (1981), using an iterative approach in spherical 
geometry, predicted a great enhancement of radiant energy at the nucleus surface due to multiple scattering effects, but it is difficult to benchmark against his results. Weissman and Kieffer (1981) also found this enhancement working in a plane-parallel geometry, multiple scattering framework. Marconi and Mendis' coma model (1984. 1986) included an adapted form of Wilson and Sen's (1980) generalized Eddington scheme to calculate the radiation field along the Sun-comet axis. They used the subsolarpoint intensity values for radiative input to the entire nucleus and predicted significant effects due to radiative transfer, in particular a strong coupling between thermal radiation from coma dust and coma gas. The dramatic radiative transfer effects predicted by these studies were due at least in part to prediction of coma optical depths much greater than those observed at Halley by Giotto imaging, and to the assumption that coma dust emission occurred uniformly over the nucleus surface rather than in a few concentrated jets on the sunward side. The coma model of Squyres et al. (1985) included an approximation for single scattering; they predicted an optically thin coma, and no dramatic radiative transfer effects were reported.

Two recent studies focused exclusively on the accurate numerical solution to the problem of light scattering in a spherically symmetric dust coma. Herman and Salo (1987) examined a variety of techniques and approximations, comparing single scattering, a low-order moment approach for isotropic multiple scattering, and a planeparallel approximation. Their results predict decreases in radiative input to the nucleus due to transport effects. Salo (1988) developed a Monte Carlo simulation of anisotropic multiple scattering in a spherical coma, again finding a moderate decrease in radiative input to the nucleus by radiative transport effects. Both studies predicted a significant redistribution of flux from the dayside of the nucleus to the nightside.

Image interpretation studies generally assume all optical depths are extremely small and make approximations accordingly. Recent image studies have focused on an explanation for the deviations of intensity from the $r^{-1}$ profile expected for uniform outflow, as observed by Giotto HMC. Heubner et al. (1988) studied effects of the geometry of dust emission at the nucleus surface and its manifestation in dust column densities along a line of sight. Thomas and Keller (1990) performed an exhaustive study analyzing the Giotto HMC images. They applied Gauss' law in their image interpretation to argue that the observed intensity profiles may indicate particle fragmentation near the nucleus. The latter two studies work within an "unattenuated single scattering" approximation in our formulation (i.e., single scattering with all attenuating optical depths neglected, see below). Jewitt (1991) surveyed photometric coma analysis and included a presentation on viewing geometry effects in coma images. Sekanina (1987) has related simulated images of large-scale coma dust patterns to idealized nucleus sources, varying nucleus rotation states, and viewing geometry. In an earlier study which considered multiple scattering, Mukai et al. (1985) calculated the multiple scattering of sunlight near the nucleus surface in plane parallel geometry, and related optical depths and surface albedo to reflected and transmitted intensity in the coma.

Along more theoretical lines, Shia and Yung (1986), using a variational approach, presented some analytic results for light scattering in a spherical atmosphere with proposed applicability to comets. Germogenova et al. (1969) performed a numerical calculation of the general problem of light scattering in spherical shell geometry with parallel illumination for the terrestrial atmosphere based on a successive approximation technique. Few details on the numerical scheme are given in their article and they show results only for single-scattered light, but their approach is apparently similar to ours.

Our study is undertaken in an attempt to overcome three major deficiencies in previous work. First, past studies of the radiative transfer of energy in the coma have assumed that the spatial distribution of dust in the coma varies only in the radial direction (spherical symmetry). Under extremely active conditions Comet Halley may have had an approximately spherically symmetric coma but it appears that a more realistic model is an optically thin spherically symmetric background coma (optical depth measured perpendicular to the surface of a few hundredths) with more optically substantial jets (optical depth measured through the axis of the jet of a few tenths up to unity for a major outburst of dust). These optical depths are collected or inferred from results of Sen $e t a l$. (1990), Keller et al. (1987) and Mukai et al. (1985).

Second, image interpretation studies apply approximations which strictly limit solution validity to tenuous single scattering conditions. Sen et al. (1990) reported observations of Halley which show a lack of polarization near the nucleus; they suggest this may be due to multiple scattering effects by fresh dust ejecta. Metz and Haefner (1987) also interpret their March 1986 observations of circular polarization at Comet Halley as indicating that multiple scattering prevailed near the nucleus at the time of measurement. Less approximate numerical solutions which include multiple scattering have in the past been tailored to optically deep cometary atmospheres. Such treatments for comas of great optical depth typically involve low-order moment approaches with a diffusion-type equation for the mean intensity. It is of general interest to develop an efficient numerical treatment for a spherical atmosphere of intermediate ( 0.1 to 1$)$ optical depth, which would allow for multiple scattering.

Third, it would be useful for image interpretation to be 


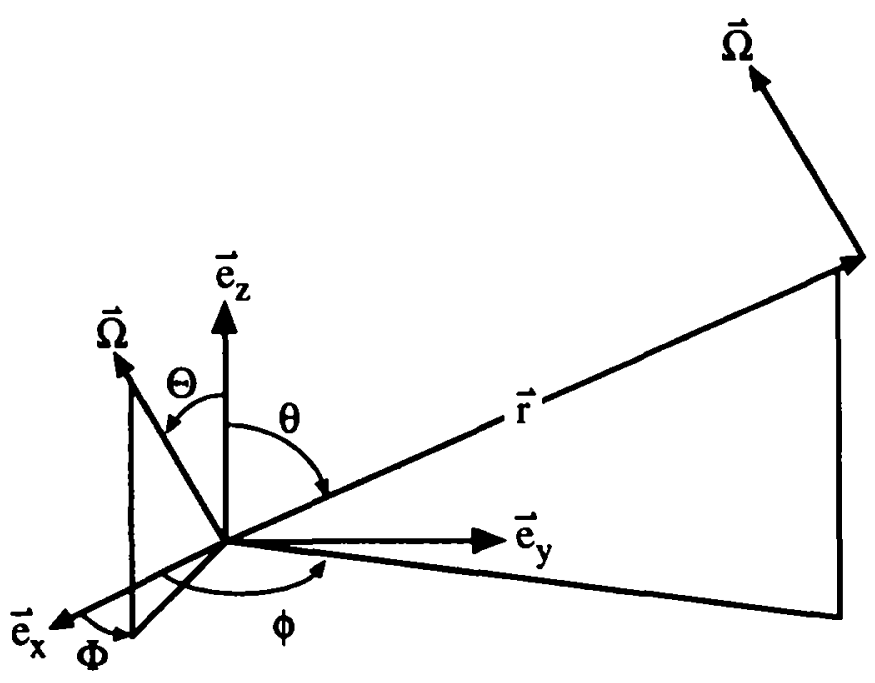

FIG. 1. The spherical coordinate system. Angles and azimuths are shown relative to the Cartesian reference. Direction is $\Omega(\Theta, \Phi)$; position is $\mathbf{r}(r, \theta, \phi)$.

able to simulate images of the entire coma. The simulation should allow for specification of optical properties throughout the coma, and for anisotropic scattering of light as would be typical of $\mu \mathrm{m}$-sized dust particles; also, the simulation should permit arbitrary viewing geometry.

We have thus developed a numerical solution to the radiative transfer equation for the anisotropic multiple scattering of visible light in a spherical shell coma, both for a spherically symmetric dust distribution and for an axisymmetric dust jet (or "active region") superposed over a tenuous spherically symmetric background. In this first stage of our work we will present simulations of the incident radiative flux at the nucleus, patterns of the mean intensity throughout the coma, and finally an approximation for the flux of thermal energy reradiated by coma dust.

\section{PHYSICAL ASSUMPTIONS FOR MODEL COMA AND MATHEMATICAL FORMULATION}

We treat radiative transfer in spherical geometry. The coordinate system is shown in Fig. 1. Spatial position is given by $\mathbf{r}(r, \theta, \phi)$ and direction by $\Omega(\Theta, \Phi)$. Parallel solar rays are incident in the direction $\Omega_{0}(\Theta=\pi, \Phi$ arbitrary), which corresponds to $-\mathbf{e}_{z}$ in Fig. 1 . The comet nucleus is at the origin and has radius $R$. Lenoble and Sekera (1961) provide a detailed discussion of coordinate systems convenient for spherical atmospheres with parallel illumination.

Our physical parametrization for light scattering in a dusty atmosphere follows that of Sobolev (1975). It is assumed that light scattering can be parametrized in terms of extinction, $\alpha$, single scatter albedo, $\lambda$, and phase function, $P$. Extinction, $\alpha$, describes the fraction of photons which interact with the host medium per unit path length; it is related to host medium number density, $n$, total cross section (for scattering and absorption), $\sigma$, the host medium mass density, $\rho$, and mass per host medium particle, $m$, by the relations $\alpha=n \sigma=\rho \sigma / m$. For a given number of photons which interact with the host medium in a unit volume of space, the single scatter albedo $\lambda$ gives the fraction which are scattered, and 1 - $\lambda$ gives the fraction which are absorbed. The phase function describes the probability that a photon will be scattered from a unit solid angle about the incident direction $\Omega^{\prime}$, into a unit solid angle about the outgoing direction $\Omega$. All three parameters are taken to represent effective values for a distribution of dust of varying composition and size. It is also assumed that all three parameters are averaged over wavelength.

If there is uniform, constant radial outflow of dust over the entire surface of a spherical nucleus, or equivalently from a point source at the origin, the dust extinction varies as $r^{-2}$ from the center of the comet. Following standard treatment for cometary problems, we note that the steady state continuity equation is

$$
\nabla \cdot \rho \mathbf{v}=q \delta(\mathbf{r})
$$

where $\mathbf{v}$ is the velocity and $q$ is the source strength (in mass per second). Integrating,

$$
\rho(r)=\frac{q}{4 \pi v r^{2}}=\frac{R^{2} \rho(r=R)}{r^{2}}
$$

where $R$ is the comet nucleus radius and $v$ is the radial speed. Using the above relation for the extinction and the host medium mass density,

$$
\alpha(r)=\frac{R^{2} \alpha(r=R)}{r^{2}}
$$

The optical depth from the Sun to the subsolar point, $\theta=$ 0 , is

$$
\tau_{\mathrm{o}}=\int_{R}^{\infty} \alpha(r) d r
$$

As noted by Herman and Salo (1987), it is convenient to use this characteristic optical depth instead of $\alpha(r=R)$ in the expression for extinction:

$$
\alpha(r)=\frac{R \tau_{\mathrm{o}}}{r^{2}}
$$


This idealized extinction profile is useful since it allows for analytic evaluation of all optical depths appearing in the problem, and is thought to be a realistic approximation of the background and asymptotic $(r \rightarrow \infty)$ dust distribution around a real comet nucleus.

For the initial development of the code an axisymmetric jet at the subsolar point is simulated in an ad hoc manner (Kitamura 1986) by assuming a Gaussian profile of the form

$$
\alpha(r, \theta)=\frac{R \tau_{\mathrm{o}}}{r^{2}}\left((X-1) e^{-\left(\theta / \theta_{\mathrm{o}}\right)^{2}}+1\right),
$$

where $X$ parametrizes the strength of the jet $(X \geq 1)$, and $\theta_{0}$ its width about the subsolar point. Note $X=1$ is the spherically symmetric case. This parametrization merely superposes Gaussian angular variation over a radial pointsource dust distribution. For purposes of image interpretation our formulation would also permit an axisymmetric cone of emission located at the surface.

The scattering phase function is parametrized by the Henyey-Greenstein phase function

$$
\boldsymbol{P}\left(\boldsymbol{\Omega} \cdot \boldsymbol{\Omega}^{\prime}\right)=\frac{1-g^{2}}{\left(1+g^{2}-2 g \boldsymbol{\Omega} \cdot \boldsymbol{\Omega}^{\prime}\right)^{3 / 2}} .
$$

where $g$ is a parameter $(0 \leq g<1)$ characterizing the forward peaking of the phase function with scattering angle: $g=0$ is isotropic scattering, and $g \rightarrow 1$ is complete forward scattering. Light is incident from direction $\boldsymbol{\Omega}^{\prime}$ and outgoing light has direction $\boldsymbol{\Omega}$, so $\boldsymbol{\Omega} \cdot \boldsymbol{\Omega}^{\prime}$ is the cosine of the scattering angle. Typical cometary dust particles should be strong forward scatterers of light (cf. Ney and Merrill 1976); realistic values of $g$ may be 0.5 and greater. Our formulation allows for $g$ and $\lambda$ to vary with position in the coma, but in all numerical results shown here they are taken to be constant.

We assume that parallel solar rays are incident from the sunward direction as $r$ tends to infinity; this boundary condition for the specific intensity is described by $I(r \rightarrow \infty, \boldsymbol{\Omega})=S \pi \delta_{2}\left(\boldsymbol{\Omega} \cdot \boldsymbol{\Omega}_{\mathrm{o}}\right)$ for $0 \leq \theta \leq \pi / 2$ and all $\phi$; and where $\pi S$ is the incident solar flux, $\delta_{2}$ is a twodimensional delta function, and $\boldsymbol{\Omega}_{\mathrm{o}}(\Theta=\pi, \Phi$ arbitrary $)$ is the direction of the incident rays. We will assume the atmosphere can be truncated at some distance $r=r_{\max }$, transforming a spherical envelope domain to a spherical shell. (For the results in this paper we have set $r_{\max }=35$ comet radii.) The nucleus' albedo is assumed to be 0 , corresponding to no reflection of light at the comet surface. The model is summarized schematically in Fig. 2 .

It is convenient to work with an integral form of the radiative transfer equation. Let us define a path length $s_{\mathrm{b}}$ as the distance such that $\mathbf{r}-s_{\mathrm{b}} \boldsymbol{\Omega}$ extends from position

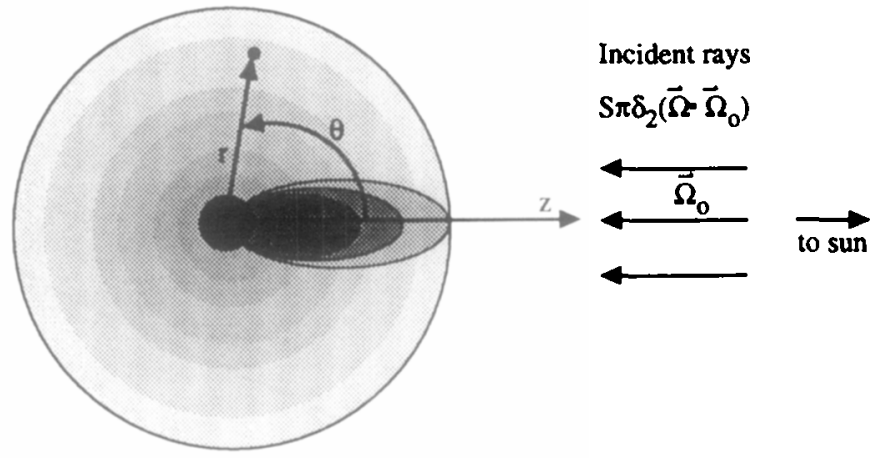

FIG. 2. Schematic diagram of the physical model.

$r$ to the boundary of the domain (either the nucleus surface or the top of the atmosphere):

$$
s_{b}(\mathbf{r}, \mathbf{\Omega})=\left\{\begin{array}{r}
\mathbf{r} \cdot \mathbf{\Omega}-\sqrt{(\mathbf{r} \cdot \boldsymbol{\Omega})^{2}-r^{2}+R^{2}} \\
\mathbf{r} \cdot \boldsymbol{\Omega}+\sqrt{(\mathbf{r} \cdot \mathbf{\Omega})^{2}-r^{2}+r_{\max }^{2}} \\
\text { if } \mathbf{r} \cdot \mathbf{\Omega}-\sqrt{r^{2}-R^{2}} \geq 0 \\
\text { if } \mathbf{r} \cdot \mathbf{\Omega}-\sqrt{r^{2}-R^{2}}<0
\end{array}\right\} .
$$

Following the formulation of Case and Zweifel (1967, Section 3.6), we have the following expression for the specific intensity of light, $I(\mathbf{r}, \Omega)$, outside the shadow antisunward of the nucleus, i.e., for $(\theta \leq \pi / 2)$ or $((\theta>\pi / 2)$ and $(r \sin$ $\theta>R)$ ):

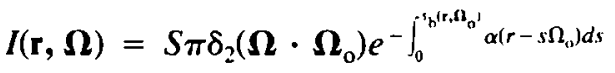

$$
\begin{aligned}
& +\int_{0}^{s_{\mathrm{b}}(\mathbf{r}, \boldsymbol{\Omega})} d s e^{-\int_{0}^{s} \alpha\left(\mathbf{r}-s^{\prime} \boldsymbol{\Omega}\right) d s^{\prime}} \frac{\alpha(\mathbf{r}-s \boldsymbol{\Omega}) \lambda}{4 \pi} \\
& \oint_{4 \pi} d \Omega^{\prime} P\left(\boldsymbol{\Omega} \cdot \mathbf{\Omega}^{\prime}\right) I\left(\mathbf{r}-s \mathbf{\Omega}, \mathbf{\Omega}^{\prime}\right) .
\end{aligned}
$$

This expression physically represents an integral of source terms along a line of sight in the direction $-\boldsymbol{\Omega}$. The first term on the right hand side represents attenuated, unscattered parallel solar rays, which may be thought of as a source located on the sunward edge of the domain. The second term on the right hand side represents scattered light arriving along the line of sight. For positions in the shadow, i.e., $((\theta>\pi / 2)$ and $(r \sin \theta \leq R))$, direct solar rays are obscured by the nucleus and the first term on the right hand side is absent.

Again following the analysis of Case and Zweifel (1967, chapter 2), or simply by observation, the solution is invariant for an arbitrary rotation about the $z$-axis. Thus we will discretize in $(r, \theta)$ and define discrete ordinates for discretization in $(\Theta, \Phi)$, but azimuthal spatial dependence, $\phi$, never explicitly appears in the problem. A discussion 
of general symmetry properties of the transport equation is made by Case and Zweifel (1967), and note of the symmetry for this problem was made by Lenoble and Sekera (1961).

\section{NUMERICAL SOLUTION}

Several aspects of the problem pose difficulties for numerical solution. The incident parallel rays are described by a delta function, which introduces a singular direction dependence in the solution, and the direction dependence of the scattered radiation field will to some extent bear the imprint of this delta-function source.

The obstacle presented by the nucleus yields discontinuities in the solution related to two effects. First, the specific intensity is discontinuous between the sunlit portion of the coma and the cylinder swept out by the antisunward shadow. Second, the solution is directionally discontinuous when paths of integration are tangent to the limb of the nucleus.

As an additional difficulty, the density of dust scattering sites drops off rapidly away from the nucleus, and the intensity of scattered light follows this drop off. Thus distant from the nucleus, scattered light from near the nucleus tends to behave as a point source illuminating the distant position (scattered light illuminating the position $\mathbf{r}$ arrives primarily along directions whose integration paths pass near the nucleus), and this gives rise to abrupt variation in direction dependence distant from the nucleus.

To cope with these problems, following Larsen (1988), we decompose the solution to the radiative transfer equation into terms representing photons which have undergone successively higher numbers of scattering events: $I=I_{0}+I_{1}+I_{\geq 2}$, so that $I_{0}$ represents unscattered photons, $I_{1}$ single-scattered photons, and $I_{\geq 2}$ photons which have undergone two or more scatterings. Removing the direct component $I_{0}$ separates the delta-function component from the solution and is standard treatment for atmospheric problems. Removing the single-scattered term serves two purposes. First, the first spatial derivative of $I_{1}$ is discontinuous across the shadow, and removing this component leaves us with a solution that is continuous across the shadow up to the second spatial derivative. Second, while far from the nucleus $I_{\geq 2}$ will still develop an abruptly varying direction dependence due to the point source appearance of scattered light from near the nucleus, $I_{\geq 2}$ is a relatively dim component of the radiation field at distances far from the nucleus. Thus for a numerical solution to $I_{\geq 2}$, it is expected that we can truncate the spherical shell atmosphere before $I_{\geq 2}$ develops its abrupt variation in direction.

We can find analytic expressions for $I_{0}$ and $I_{1}$ (which require some involved but straightforward numerical evaluation), and since $I_{\geq 2}$ is a smoothed-out and relatively well-behaved function, numerical solution for $I_{\geq 2}$ alone is much easier than it would be for $I_{0}+I_{1}+I_{\geq 2}$ or $I_{1}+I_{\geq 2}$. We are left with two problems. First, all components of scattering are discontinuous in direction when their corresponding paths of integration are tangent to the limb of the nucleus. Second, separating the unscattered component removes the singular delta-function nature in regions where unscattered light is the dominant component in the radiation field, namely at shallow optical depths on the sunward side of the coma; however, when the phase function is strongly forward-peaked, the abrupt variation in direction dependence associated with the delta-function incident rays is retained over many scatterings and deeper into the atmosphere in the sunlit portion of the coma. We partially overcome these problems with a judicious choice of direction ordinates.

The unscattered component is

$$
\begin{aligned}
& I_{0}(\mathbf{r}, \boldsymbol{\Omega})=\left\{\begin{array}{l}
S \pi \delta_{2}\left(\boldsymbol{\Omega} \cdot \boldsymbol{\Omega}_{0}\right) e^{-\int_{0}^{s_{\mathrm{b}}\left(r, \Omega_{\mathrm{o}^{\prime}}\right.} \alpha\left(\mathbf{r}-s \boldsymbol{\Omega}_{\mathrm{o}}\right) d s} \\
0
\end{array}\right. \\
& \left.\begin{array}{l}
\text { if }(\theta \leq \pi / 2) \operatorname{or}((\theta>\pi / 2) \text { and }(r \sin \theta>R)) \\
\text { otherwise, i.e., }(\theta>\pi / 2) \text { and }(r \sin \theta \leq R)
\end{array}\right\}
\end{aligned}
$$

Physically, this expression represents incident rays multiplied by line-of-sight transmission, with the comet nucleus casting a shadow in the antisunward direction. For a fixed direction, the unscattered component is spatially discontinuous across the antisunward shadow line and is singular in direction due to the delta function representing the incident radiation. Once the optical depth appearing in the above expression is evaluated, either analytically or numerically, the unscattered component is known.

The single-scattered component is

$$
\begin{aligned}
& I_{1}(\mathbf{r}, \boldsymbol{\Omega})=\int_{0}^{s_{\mathrm{b}}(\mathbf{r}, \boldsymbol{\Omega})} d s e^{-\int_{0}^{1} \alpha\left(r-s^{-} \mathbf{\Omega} d s .\right.} \frac{\alpha(\mathbf{r}-s \boldsymbol{\Omega}) \lambda}{4 \pi} \\
& \oint_{4 \pi} d \Omega^{\prime} P\left(\Omega \cdot \Omega^{\prime}\right) I_{0}\left(\mathbf{r}-s \Omega, \Omega^{\prime}\right)
\end{aligned}
$$

For a fixed position, the single-scattered component is discontinuous in direction when the path of integration in Eq. (11) crosses the limb of the nucleus, and for a fixed direction the single-scattered component has a discontinuous first spatial derivative across the antisunward shadow line due to path integration of the spatially discontinuous unscattered component. Assuming optical depths can be determined, the single-scattered term is formally solved at this point since it can be evaluated in terms of the known unscattered component.

To find the light scattered two or more lines, let us 
define the following iterative scheme (Neumann solution or lambda iteration; see Case et al., 1953, pp. 45-46):

$$
\begin{aligned}
& I_{\geq 2}^{k+1}(\mathbf{r}, \boldsymbol{\Omega})=\int_{0}^{s_{b}(\mathbf{r}, \boldsymbol{\Omega})} d s e^{-\int_{0}^{s} \alpha\left(\mathbf{r}-s^{\prime} \mathbf{\Omega}\right) d s^{\prime}} \frac{\alpha(\mathbf{r}-s \boldsymbol{\Omega}) \lambda}{4 \pi} \\
& \oint_{4 \pi} d \boldsymbol{\Omega}^{\prime} P\left(\boldsymbol{\Omega} \cdot \boldsymbol{\Omega}^{\prime}\right)\left(I_{\geq 2}^{k}\left(\mathbf{r}-s \boldsymbol{\Omega}, \boldsymbol{\Omega}^{\prime}\right)+I_{1}\left(\mathbf{r}-s \boldsymbol{\Omega}, \boldsymbol{\Omega}^{\prime}\right)\right),
\end{aligned}
$$

where for the first step, $k=0$, we set $I_{\geq 2}^{0}=0$. The component $I_{\geq 2}$ has a discontinuous second derivative in space across the shadow line (from integration of the firstorder spatially discontinuous $I_{1}$ term), and $I_{\geq 2}$ is stepwise discontinuous in direction across the limb of the nucleus.

To carry out the solution numerically, we discretize in position and define discrete ordinates in direction. Radial position, $r$, is discretized roughly evenly on a logarithmic scale, see Fig. 11; position $\theta$ is discretized either evenly over $[0, \pi]$ or with points concentrated near the subsolar point if a jet is present. The direction ordinates are defined so that quadrature is consistent with the limb direction discontinuity mentioned. We first evaluate Eq. (10) for $I_{0}$ at each position grid point $\left(r_{j}, \theta_{i}\right)$ and in each direction ordinate $\left(\Theta_{n}, \Phi_{m}\right)$, and then repeat the process for $\mathrm{Eq}$. (11) using a library Romberg integration scheme.

With this solution for $I_{1}$, we proceed with the lambda iteration scheme for $I_{\geq 2}$. For each iteration step, we numerically evaluate Eq. (12) for each position grid point $\left(r_{j}, \theta_{i}\right)$ and in each direction ordinate $\left(\Theta_{n}, \Phi_{m}\right)$. To perform the path integration, we (1) determine where the path crosses the mesh defined by the $r-\theta$ discretization, (2) interpolate the values of the integrand at these crossing points, and then (3) assume that the specific intensity varies linearly between crossing points and apply trapezoidal rule integration. Details on the path integration scheme are given in Appendix 1. The direction integral appearing in Eq. (12) is performed by quadrature. Specification of discrete ordinates and the quadrature scheme is discussed in Appendix 2.

The number of iterations required for convergence should approximately equal the average number of scatterings a photon makes between entry and exit from the spherical shell atmosphere (Case et al. 1953, p. 46). Convergence of $I_{\geq 2}^{k}$ to the true solution will depend on three factors: one, sufficient direction resolution for accurate evaluation of the direction integral appearing on the right hand side of Eq. (12); two, sufficient spatial resolution for accurate evaluation of the path integral appearing in Eq. (12); and three, truncation of the domain, which introduces a singularity in direction dependence at the top of the atmosphere (see Cheyney and Arking 1976). This should be a small effect since we can extend the upper limit until any effects of the singularity are negligible.
For the standard test case we discuss later, a spherically symmetric coma with $\tau_{\mathrm{o}}=1, \lambda=1$, and $g=0$, the solution converged in four to five iterations, implying that photons typically scatter four or five times between entry and exit from the coma. Low resolution runs were performed on Sun and IBM workstations, and final runs were done on a Cray Y-MP. Run time was on the order of $1 \mathrm{hr}$ cpu on the Cray computer.

\section{RESULTS}

First, we present calculations of the radiant flux of visible light impinging on the nucleus surface. We benchmark our calculation of the flux against previous work done for the case of a spherically symmetric coma and then discuss the case of a dust jet. Second, we calculate the mean intensity of light throughout the coma. Finally we use our calculation of the mean intensity of visible light to examine an approximate solution for the flux impinging at the nucleus surface due to thermal radiation emitted by dust in the coma.

\section{4a. Flux of Visible Light Impinging on the Nucleus Surface}

The flux of radiant energy impinging on the nucleus drives the sublimation of gas, the subsequent entrainment of dust, and the formation of the coma (cf. Gombosi et al. 1986). Since the bulk of the sun's radiant energy is transported at visible wavelengths, a model of nucleus activity should include a realistic estimate of the visible light striking it. The problem is potentially complicated because the presence of ejected dust may modify the radiation field at the nucleus surface. Absorption of light by dust will diminish the visible-wavelength energy available to drive sublimation, and scattering may channel light to the nucleus. At this present time we cannot model the complicated time dependence inherent in such feedback processes, but for a given spatial dust distribution we can investigate the intensity of radiation at the nucleus to determine if feedback between the ejected dust and the nucleus source may be significant.

The radial component of flux may be thought of as the net upward flux, in a local direction reference, for a given position. For visible light, let us label the radial component

$$
F_{\mathrm{vis}}(r, \theta)=\oint_{4 \pi} d \mathbf{\Omega}\left(I_{0}+I_{1}+I_{\geq 2}\right) \mathbf{\Omega} \cdot \mathbf{e}_{\mathrm{r}}
$$

By plugging in the relation for the unscattered specific intensity, $I_{0}$, given by Eq. (10), the direct unscattered component of the radial flux is 


$$
F_{\text {vis(dir) }}=\left\{\begin{array}{ll}
-S \pi \cos \theta e^{-\tau\left(\mathbf{r}, \Omega_{0}, s_{\mathrm{b}}\left(\mathrm{r}, \Omega_{\mathrm{o}}\right)\right)} & \text { if } \theta \leq \pi / 2 \\
0 & \text { if } \theta>\pi / 2
\end{array}\right\}
$$

where the optical depth

$$
\tau(\mathbf{r}, \mathbf{\Omega}, s)=\int_{0}^{s} d s^{\prime} \alpha\left(\mathbf{r}-s^{\prime} \mathbf{\Omega}\right)
$$

has been introduced.The single-scattered component of the radial flux is

$$
F_{\text {vis(ss) }}(r, \theta)=\oint_{4 \pi} d \Omega I_{1} \mathbf{\Omega} \cdot \mathbf{e}_{\mathrm{r}}
$$

For comparison to past work, we take "multiple scattering" to mean "one or more scatterings." The multiply scattered component of the radial flux is then

$$
F_{\text {vis(ms) }}(r, \theta)=\oint_{4 \pi} d \mathbf{\Omega}\left(I_{1}+I_{\geq_{2}}\right) \mathbf{\Omega} \cdot \mathbf{e}_{r} \cdot
$$

The net downward flux of visible radiation at the surface is equal to the negative of the radial flux evaluated at the surface. Since we have taken the nucleus albedo to be zero there is no upward reflected light at the surface.

For optically tenuous conditions, the single scattering term, $I_{1}$, is a sufficient approximation for the visible radiation field. Herman and Salo (1987) numerically evaluated an expression for the single-scattered flux of light at the nucleus surface, averaged over the surface, with zero surface albedo:

$$
\bar{F}_{\mathrm{vis}(\mathrm{ss})}=\frac{1}{4 \pi R^{2}} \int_{0}^{2 \pi} R d \phi \int_{0}^{\pi} R d \theta \sin \theta F_{\mathrm{vis}(\mathrm{ss})}(r=R, \theta)
$$

for the case of a spherically symmetric coma. They used the expression both for coma simulation and as a benchmark reference to check other numerical techniques. As a check on our computer code, we compute this quantity for a succession of Sun-to-subsolar point optical depths, $\tau_{\mathrm{o}}$, with an isotropic phase function $(g=0)$, and compare with their results in Fig. 3. As the coma is loaded with more dust there is more scattered light at the surface. We essentially match their values, although our values are slightly greater at larger optical depths.

Salo (1988) pursued a Monte Carlo treatment of the radiant flux incident on the nucleus surface for the case of a spherically symmetric coma, and presented the flux of multiply scattered visible light impinging on the nucleus surface, $F_{\text {vis(ms) }}(r=R, \theta)$, for a succession of values of the Henyey-Greenstein phase function parameter, $g$. As $g$ increases from 0 to 1 , light is more strongly forward

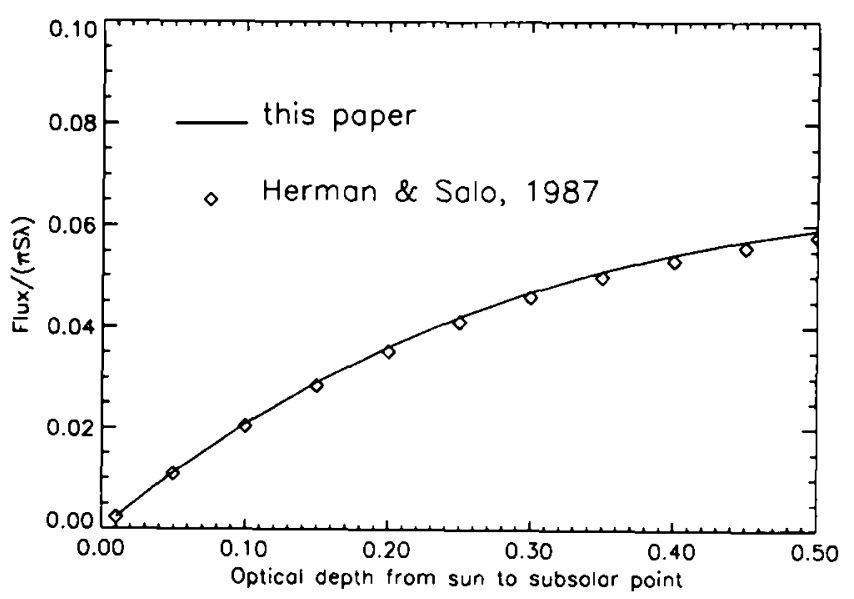

FIG. 3. Net downward single-scattered flux of visible radiation at the nucleus surface averaged over the nucleus surface, $\bar{F}_{\text {vis(s) }}$, for a spherically symmetric coma, in function of increasing the Sun-tosubsolar point optical depth, $\tau_{0}$.

scattered, and the scattered flux penetrating to the surface increases. To check our multiple scattering solution, we evaluate $F_{\text {vis(ms) }}(r=R, \theta)$ for three phase functions $(g=$ $0, g=0.5$, and $g=0.75$ ) and compare to the results of Salo's Monte Carlo simulation in Fig. 4. The coma is spherically symmetric, the single scatter albedo is $\lambda=1$, and the Sun-to-subsolar point optical depth is $\tau_{\mathrm{o}}=1$. The agreement appears to be quite good. (Comparison values for the case $g=0$ were taken from Salo's Table II, but comparison values for $g=0.5$ and $g=0.75$ were read "by eye" from Salo's Fig. 3b.)

Overall the coma may be optically tenuous, but dust emission appears to be from isolated cracks or "hot spots," possibly consisting of bare ice; these hot spots
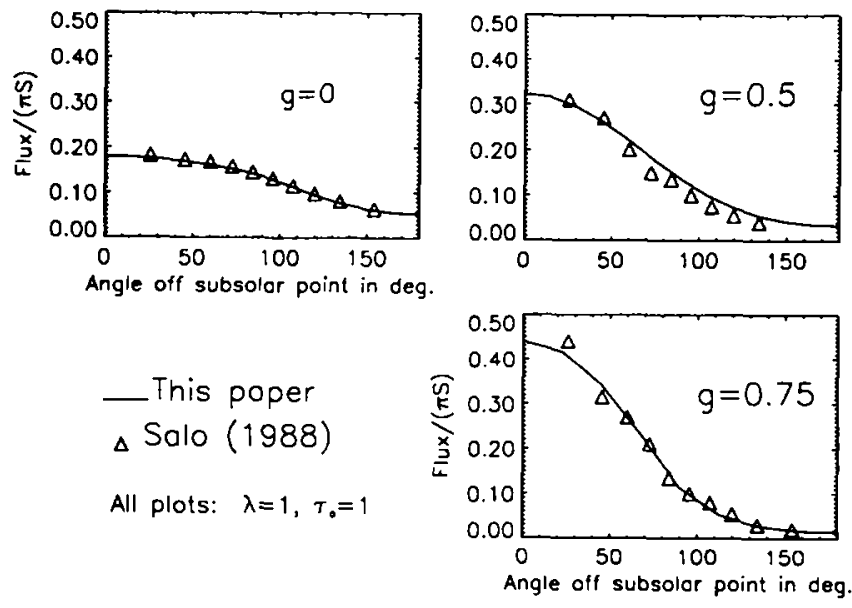

FIG. 4. Downward multiply scattered flux of visible radiation at the nucleus surface, $F_{\text {vis(ms) }}(r=R, \theta)$, with increasing forward scattering, for a spherically symmetric coma. 

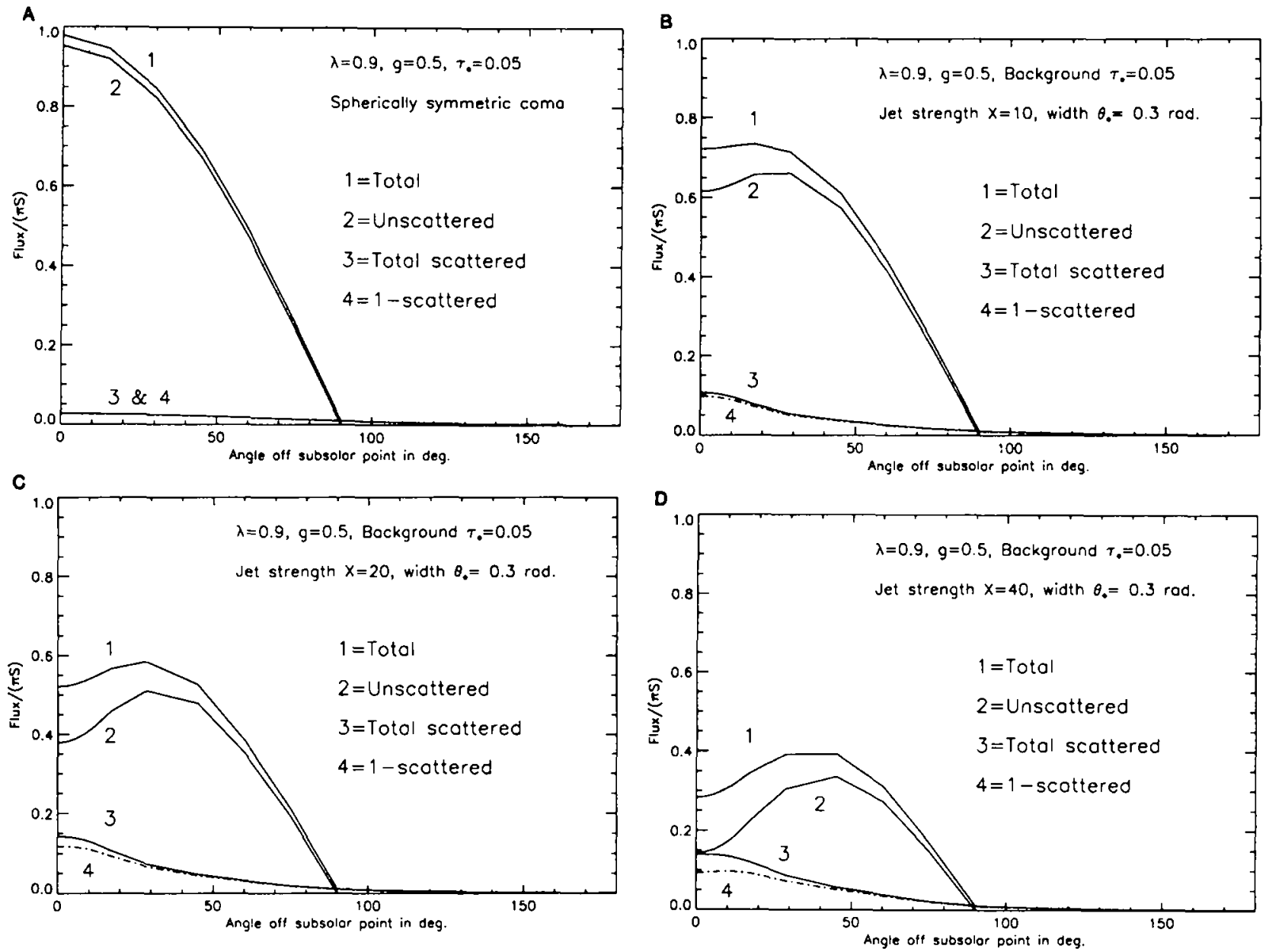

FIG. 5. Downward flux of multiply scattered light at the nucleus surface for a coma with an axisymmetric jet of increasing strength.

may be quite sensitive to visible radiation. Since the sources of dust emission have optically substantial dust jets extending above them, scattering effects within the dust jet may affect energy deposition at the hot spot. In Fig. 5 we investigate the extent to which attenuation and scattering within jets of increasing strength might affect the flux of visible light at the base of the jet. This panel compares the direct, single-scattered, and multiply scattered downward net fluxes at the surface for jets of different strengths. The characteristic optical depth for the symmetric background coma is $\tau_{0}=0.05$, the jet angular width is $\theta_{0}=0.3$ radians, and the single scatter albedo is $\lambda=$ 0.9 for all cases. It is hard to guess a realistic value of $\lambda$, especially for a coma composed of materials of widely varying complex index of refraction (such as sooty organics, crystalline silicates, and possibly ice particles). We chose a value $\lambda$ near 1 to highlight scattering effects; a smaller value will damp out scattering effects. Our results represent approximate upper limits for effects of scattered light.

For Fig. 5a, jet strength $X=1$ represents the spherically symmetric case, and the coma is tenuous, so almost all the visible flux is transported by the direct component, $F_{\text {vis(dir) }}$; the single-scattered component $F_{\text {vis(ss) }}$ is small, and flux due to $I_{\geq 2}$ is negligible.

In Fig. 5b, jet strength $X=10$, the actual optical depth from Sun to subsolar point through the axis of the jet is $\tau=0.5$. The direct component $F_{\text {vis(dir })}$ is noticeably attenuated in the jet region, and a significant amount of flux is transported to the base of the jet (the subsolar point) by single-scattered light, but the flux due to the $I_{\geq 2}$ component is still very small.

In Fig. 5c, jet strength $X=20$, the optical depth through the axis of the jet is $\tau=1$. The direct component $F_{\text {vis(dir) }}$ is increasingly attenuated in the jet, and scattering effects channel more light to the base of the jet, with the $I_{\geq 2}$ component beginning to account for a noticeable portion of the flux.

In Fig. 5d the jet strength is $X=40$, yielding an axial optical depth through the jet of $\tau=2$. The direct component $F_{\text {vis(dir) }}$ is strongly attenuated in the jet, and the scattered flux $F_{\text {vis(ms) }}$ is greatly enhanced. The direct and scat- 

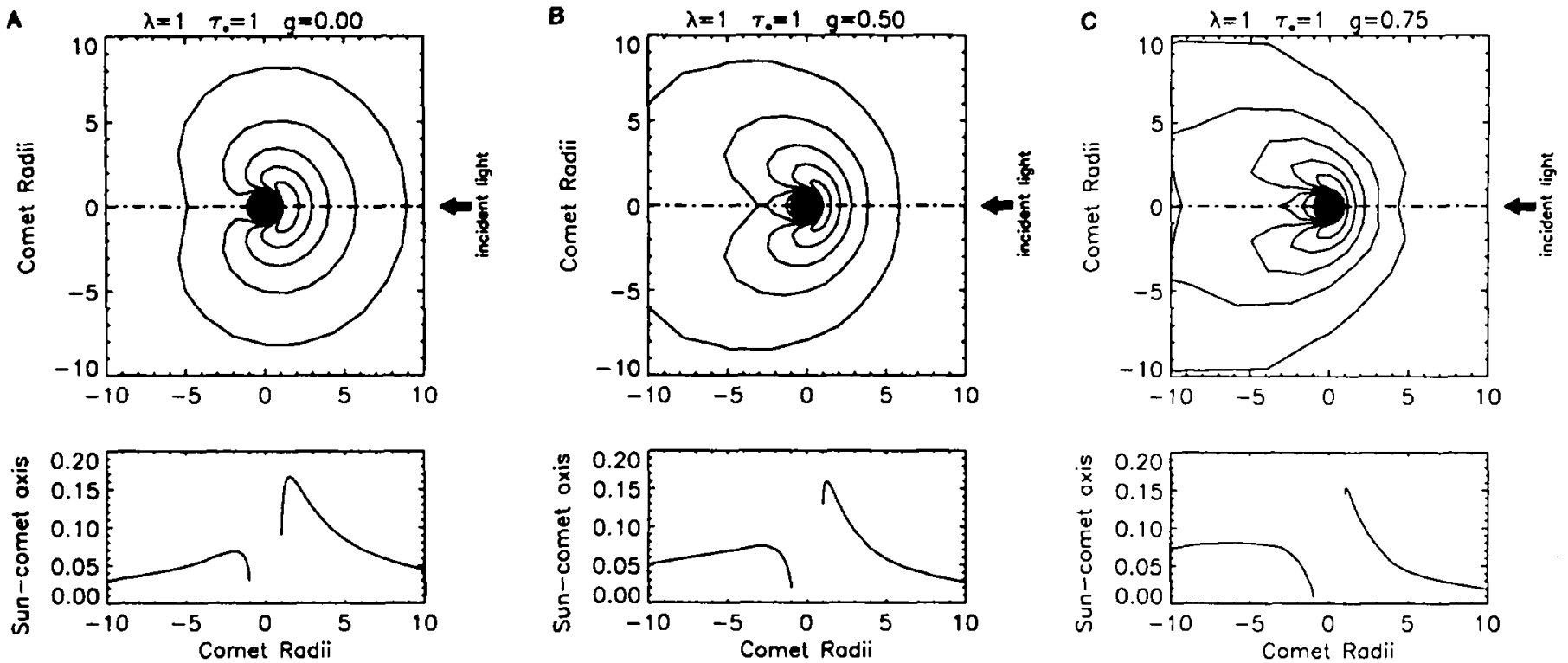

FIG. 6. Mean intensity of multiply scattered visible light, $J_{\text {visms }}(r, \theta)$, throughout a symmetric coma, for increasing forward peaking of the phase function.

tered terms are about equal at the base of the jet. The $I_{\geq 2}$ component now accounts for about one-third of the scattered flux at the base of the jet.

Hence, for the modeling of a comet nucleus' activity under conditions of a sunward jet, visible wavelength radiative input is probably well characterized by single scattering for optical depths up to about unity (measured through the axis of the jet); it is only for axial optical depths greater than 1 that $I_{\geq 2}$ accounts for significant deposition of energy in the visible. Under all conditions examined, the total visible flux never exceeds unity, i.e., the normalized visible flux at the subsolar point of a bare nucleus.

\section{4b. Mean Intensity of Multiply Scattered Visible Light throughout the Coma}

We define the mean intensity of multiply scattered light as

$$
J_{\text {vis(m) }}(r, \theta)=\frac{1}{4 \pi} \oint_{4 \pi} d \Omega\left(I_{1}+I_{\geq 2}\right)
$$

The mean intensity gives a qualitative measure of the radiation field throughout the coma. Computation of this quantity for a spherically symmetric dust coma allows us to extend the check of our code, this time against our physical intuition and (at least qualitatively) against the results of past authors. Also, by varying optical parameters in our model we get a quantification of the physical manifestations of various factors in the transport of light.

Figure 6 presents the visible mean intensity $J_{\text {vis(ms) }}$ for the case of a spherically symmetric coma and compares isotropic scattering ( $g=0$ ) and forward-peaked scattering ( $g=0.5$ and $g=0.75$ ). The Sun-to-subsolar point optical depth is $\tau_{\mathrm{o}}=1$. The pattern is qualitatively similar for smaller optical depths. Below each angular pattern is a cross-section plot of the mean intensity along the Suncomet axis. In Fig. 6a, for isotropic scattering ( $g=0$ ), the cross-section profile peaks just before the subsolar point. As the phase function becomes increasingly forward-peaked, for $g=0.5$ (Fig. 6b) and then $g=0.75$ (Fig. 6c), the scattered radiation builds up more and more abruptly at the comet surface, and increasing light is transported antisunward of the nucleus. The angular pattern is rounded and diffuse for $g=0$, whereas the pattern for the more forward peaked phase function cases is "swept past" the comet. The radial cross-section profiles are similar to the profile found by Marconi and Mendis (1984, see their Fig. 3) for an optically deeper case $\left(\tau_{\mathrm{o}}=2.9\right)$ with forward peaked scattering and a more absorptive dust coma, $\lambda=0.51$. This profile was also found by Herman and Salo (1987, see their Fig. 4) for the conditions $g=0$, $\tau_{\mathrm{o}}=2$ and 3 , and $\lambda=0.9$.

While the spherically symmetric case is useful for test purposes, it appears a more realistic model should incorporate one or several isolated sources of dust on the sunward face of the comet, with emission in the sunward direction. We approximate this with an axisymmetric dust feature at the comet subsolar point. Figure 7 shows the mean intensity patterns resulting from an increasing strength of such a dust jet. Figure 7a shows the angular pattern and Sun-comet axial cross section of mean inten- 

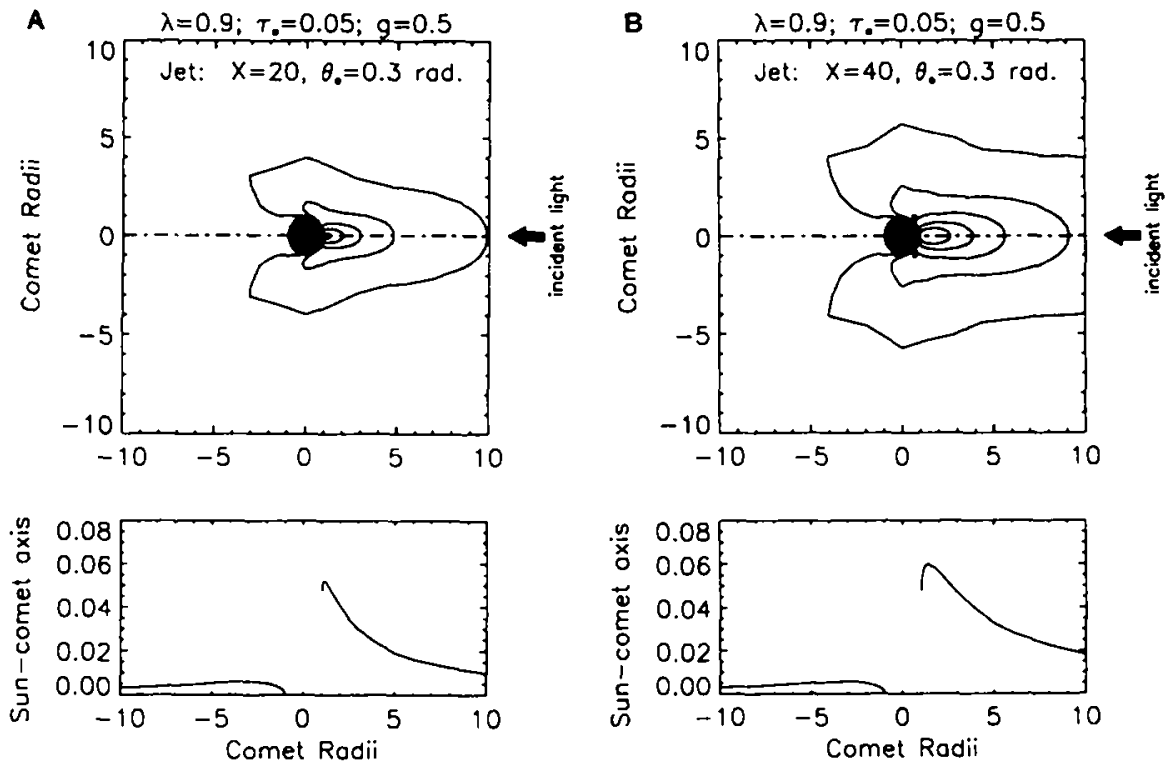

FIG. 7. Mean intensity of multiply scattered light, $J_{\text {visms } s}(r, \theta)$, throughout a coma with an axisymmetric jet at the subsolar point. In Fig. $7 \mathrm{~A}$ the optical depth through the axis of the jet is 1 . In Fig. 7B the optical depth through the axis of the jet is 2.

sity computed for a jet of strength $X=20$, background Sun-to-subsolar point optical depth $\tau_{\mathrm{o}}=\mathbf{0 . 0 5}$, and therefore an optical depth through the axis of the jet of $\tau=1$. The second case, Fig. $7 \mathrm{~b}$, is for the same background, $\tau_{\mathrm{o}}=0.05$, but a jet strength of $X=40$, so the optical depth through the axis of the jet is $\tau=2$. Comparison between the two figures demonstrates the brightening of the scattered radiation field as the coma is loaded with more dust. The jet angular width for both cases is $\theta_{0}=$ 0.3 radians; the phase function is $g=0.5$; the single scatter albedo is $\lambda=0.9$.

Thus, examination of the pattern of $J_{\text {vis(ms) }}$ throughout the coma shows the response of the radiation field to variation of optical parameters such as the phase function. It also reveals the radiative response to the spatial distribution of dust and to increased dust loading in the coma. $J_{\text {vis(ms) }}$ also serves an approximate source function for the thermal radiation reemitted by coma dust particles, as discussed in the next section.

\section{4c. Approximate Flux of Thermal Reradiated Energy Impinging on the Nucleus Surface}

Thermally reemitted energy from dust particles contributes to the radiant energy impinging on the nucleus. The visible mean intensity acts as an approximate source term for the thermal reemission of radiation by dust, and we can use our calculation of $J_{\text {vis(ms) }}$ to examine the behavior of this flux. A volume of dust in the coma absorbs a fraction $1-\lambda$ of the incident visible light. If we assume that the dust particles are in thermal equilibrium and therefore reemit all absorbed energy, and that the emission is isotropic, and further that the incident thermal energy from the Sun is zero, then the emission at a point in space is

$$
\frac{1}{4 \pi}(1-\lambda) \alpha \oint_{4 \pi} d \Omega\left(I_{0}+I_{1}+I_{\geq 2}\right)
$$

If we neglect any scattering or absorption of thermal radiation, we can integrate the above emission term along a line of sight, and obtain the specific intensity of thermal radiation. For purposes of comparison, let us decompose the downward flux of thermal energy at the nucleus surface into two components. The first is the downward flux of thermal reemission of absorbed, unscattered incident light (a "direct" term),

$$
\begin{aligned}
& F_{\text {Ir(dir) }}(\mathbf{r}(r=R, \theta))=\int_{\mathbf{\Omega}: \mathbf{\Omega} \cdot \mathbf{e}_{\mathrm{r}}<\mathbf{0}} d \boldsymbol{\Omega} \boldsymbol{\Omega} \cdot \mathbf{e}_{\mathrm{r}} \int_{0}^{s_{\mathrm{b}}(r, \Omega)} \\
& d s(1-\lambda) \frac{S}{4} e^{-\tau\left(r-s \boldsymbol{\Omega}, \boldsymbol{\Omega}_{\mathrm{o}}, s_{\mathrm{b}}\left(r-s \boldsymbol{\Omega}, \boldsymbol{\Omega}_{\mathrm{o}}\right)\right)} \alpha(\mathbf{r}-s \boldsymbol{\Omega}),
\end{aligned}
$$

and the second is the downward flux of thermal reemission of absorbed, multiply scattered light,

$$
\begin{aligned}
F_{\mathrm{rr}(\mathrm{ms})}(\mathbf{r}(r=R, \theta)) & =\int_{\mathbf{\Omega}: \mathbf{\Omega} \cdot \mathbf{e}_{\mathrm{r}}<0} d \boldsymbol{\Omega} \boldsymbol{\Omega} \cdot \mathbf{e}_{\mathrm{r}} \int_{0}^{s_{\mathrm{b}}(\mathbf{r}, \Omega)} \\
& d s(1-\lambda) J_{\mathrm{vis}(\mathrm{ms})}(\mathbf{r}-s \boldsymbol{\Omega}) \alpha(\mathbf{r}-s \boldsymbol{\Omega}) .
\end{aligned}
$$

The subscript rr refers to "reradiated" flux. The down- 


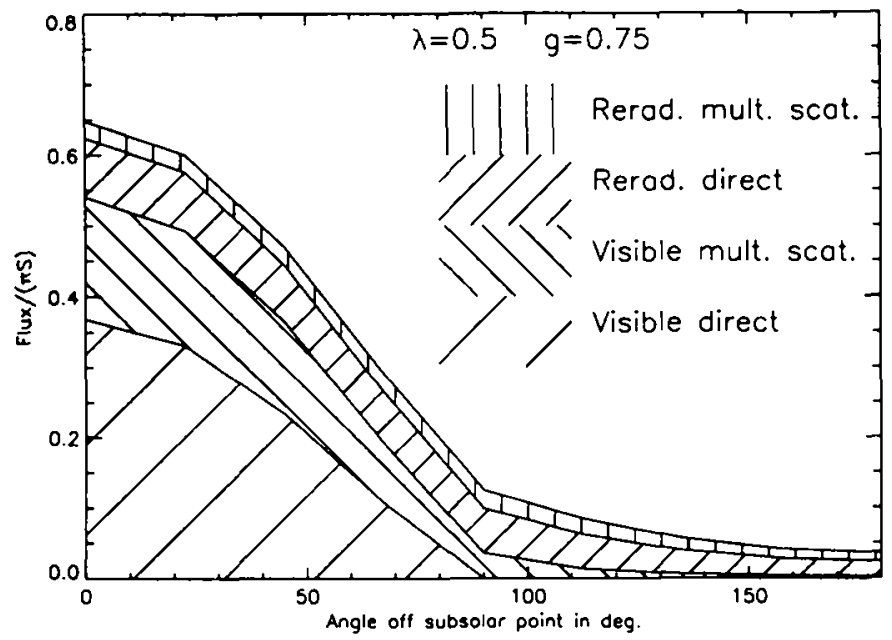

FIG. 8. Total downward flux at nucleus surface for a symmetric coma, optical depth $\tau_{0}=1$, single scatter albedo $\lambda=0.5$, and scattering parameter $g=0.75$. The various components of the total flux are also shown. (Compare to Fig. 5 in Salo's 1988 article.)

ward incident flux of thermal radiation impinging on the nucleus surface is the sum of the above two terms:

$F_{\mathrm{rr}}(r=R, \theta)=F_{\mathrm{rr}(\mathrm{dir})}(r=R, \theta)+F_{\mathrm{rr}(\mathrm{ms})}(r=R, \theta)$.

As noted by Salo (1988), this gives an upper limit to the reradiated thermal flux, since the dust is actually heated as it flows away from the nucleus and does not reradiate all energy from absorbed visible light.

First, to check our code we compute the approximate reradiated flux for a spherically symmetric coma, optical depth $\tau_{\mathrm{o}}=1$, single scatter albedo $\lambda=0.5, g=0.75$, and compare it to the results of Salo's (1988) Monte Carlo simulation. Figure 8 shows the total flux of visible and thermal energy and the separate components of which it is composed: $F_{\text {vis(dir), }} F_{\text {vis(ms). }} F_{\text {rr(dir) }}$, and $F_{\text {rr(ms) }}$. The total flux does not exceed unity (i.e., the normalized visible flux at the subsolar point of a bare nucleus). The calculation appears to be in good agreement with Salo's Fig. 5.

Figure 9 shows a comparison of $F_{\pi}(r=R, \theta)$ for a jet of width $\theta_{0}=0.3$ radians, strength $X=20$, background optical depth $\tau_{0}=0.05$, and thus a jet axial optical depth of $\tau=1$, moderate forward scattering, $g=0.5$, and varying single scatter albedo, $\lambda$. The flux of unscattered visible light, $F_{\text {vis(dir) }}$, is independent of $\lambda$, and for these jet conditions $F_{\text {vis(dir) }}$ was shown in Fig. 5c.

In Fig. 9a there is complete absorption of light, $\lambda=0$, and no scattered light; thus $F_{\text {rr(ms) }}=0$, and the reradiated flux is due completely to the direct component $F_{\text {rr(dir) }}$. In Fig. 9b the single scatter albedo is $\lambda=0.5$. The flux of visible multiply scattered light, $F_{\mathrm{vis}(\mathrm{ms})}$, is shown for reference, and the dotted line shows the fraction of reradiated flux due to the direct component, $F_{\text {rrdir) }}$. Note the contribution to the reradiated flux from multiply scattered light is negligible on the antisunward side of the nucleus. Figure 9c demonstrates high scattering conditions, $\lambda=$ 0.9 . The flux of visible light is greatly enhanced (refer to Fig. $5 c$ for these conditions) and the reradiated flux is very small. With high scattering conditions, visible energy is either scattered out of the atmosphere or absorbed by the nucleus before coma dust can absorb it, so little energy is available to be reradiated by dust.

This suggests that even for the case of an optically substantial jet (in this case the optical depth through the axis of the jet is equal to 1), the reradiated flux is quite small compared to the visible components of the radiant flux impinging on the nucleus. For the three values of $\lambda$ sampled, the reradiated flux is less than $10 \%$ of the visible flux at the subsolar point of a bare nucleus.

\section{DISCUSSION}

Keller et al. (1987) placed an upper limit of unity on optical depths observed by Giotto HMC and suggested a rough estimate of 0.28 for the maximum optical depth observed. It is generally thought that this is optically thin enough to not mediate energy flow to the nucleus. Our results indicate single scattering starts to account for a significant fraction of the incident visible flux when optical depths are several tenths, measured through the axis of the jet: our simulation shows single scattering accounts for about $15 \%$ of the incident flux of visible energy when the optical depth through the axis of the jet is 0.5. Multiple scattering of visible light appears to account for a significant portion of the budget only when optical depths through the axis of the jet reach 1 to 2 . On the other hand, even for an optically substantial jet (of axial optical depth equal to 1) the reradiated flux appears to be very small (less than 10\%) for all values of single scatter albedo, $\lambda$. For all coma conditions considered here, we find no instance of the radiant flux exceeding unity, i.e., the normalized visible flux at the subsolar point of a bare nucleus. This is an accord with studies by Herman and Salo (1987) and Salo (1988). Further, in the case of a jet, our results show little redistribution of radiant flux from the sunward face of the nucleus to the nightside, simply because there is very little dust in the antisunward portion of the coma.

During periods of greater activity than that observed by Giotto HMC, such as during the initial eruption of a jet, optical depths might be considerably larger. Apparently, violent sporadic outbursts appear to occur on comets (Festou et al. 1990, Larson et al. 1990, Lamy et al. 1989). Since the mechanism for such eruptions is not well understood, it is not inconceivable that ejected dust could play 

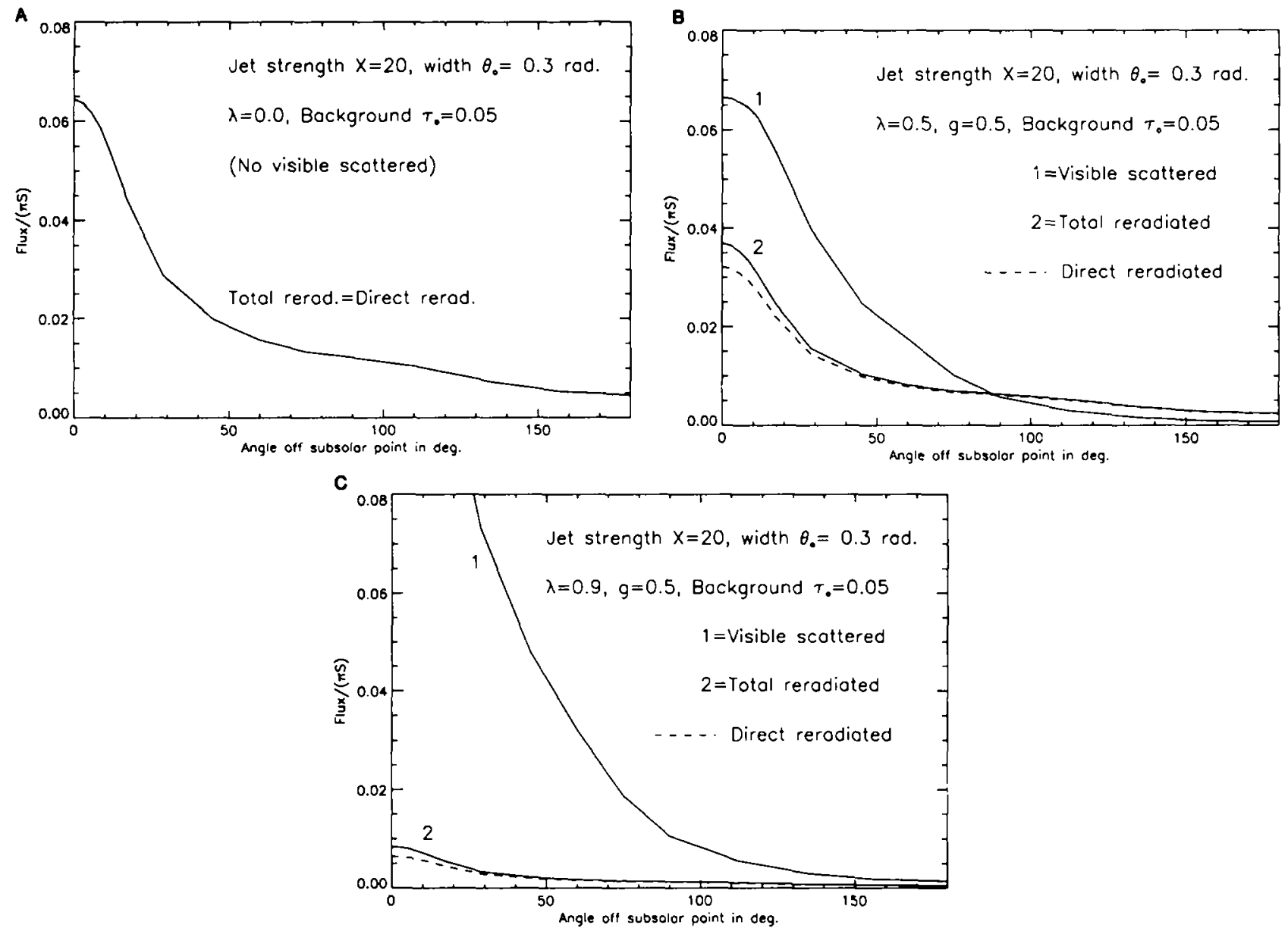

FIG. 9. Downward flux of reradiated energy at the nucleus surface for a coma with an axisymmetric jet at the subsolar point, for varying dust single scatter albedo.

some role in radiative transfer feedback to the nucleus. The most active source region on Halley appeared to be bare ice (Reitsema et al. 1989b); if the ice is semitransparent it may be sensitive to visible radiation (Kömle et al. 1990).

In view of the optical depths estimated for Giotto HMC observation conditions, it seems prudent to investigate possible multiple scattering effects for Giotto $\mathrm{HMC}$ image interpretation; furthermore, a general solution for anisotropic multiple scattering in a spherical cometary atmosphere should be useful for interpretation of high-resolution imaging of comets, especially imaging by any future comet fly-by missions.

\section{CONCLUSION}

We have developed a numerical solution for the anisotropic multiple scattering of light in a spherical shell comet atmosphere. The code has been run for a spherically symmetric coma dust distribution, benchmarked against past studies, and then run for the conditions of an axisymmetric dust jet at the subsolar point of the comet. We have investigated the radiant flux impinging on the nucleus surface and the mean intensity of light throughout the coma. The constraint of axial symmetry appears essential, since it reduces the number of independent variables in the transport equation from five to four. Within the framework of multiple scattering it would be difficult to relax the constraint of axial symmetry, at least with current computer capabilities. It is possible polarization should be taken into account for image interpretation; this is beyond the scope of our numerical treatment and would probably require a Monte Carlo approach.

The lambda iteration numerical approach has been shown to provide an efficient and accurate solution for the visible radiation field in a spherical shell atmosphere and should be quite useful for general terrestrial or planetary radiative transfer problems requiring consideration of spherical geometry.

As a next step in our work, we are developing an image 
simulation of the coma based on our solution to the radiative transfer equation. Viewing geometry (i.e., distance of observer from nucleus and phase angle with Sun) is arbitrary. We are investigating effects of coma dust optical properties and multiple scattering on images of the coma. We can simulate the azimuthal average considered by Thomas and Keller (1990) as a possible measure of particle fragmentation.

\section{APPENDIX 1. NUMERICAL PATH INTEGRATION} form

Determination of $I_{1}$ and $I_{22}$ both involve evaluation of integrals of the

$I(\mathbf{r}, \boldsymbol{\Omega})=I\left(\mathbf{r}-s_{h} \mathbf{\Omega}, \boldsymbol{\Omega}\right) e^{-\tau\left(\mathbf{r}, \boldsymbol{\Omega}, s_{h}(\mathbf{r} \mathbf{\Omega})\right)}$

$$
+\int_{0}^{s_{h}^{(r, \boldsymbol{\Omega})}} d s e^{-\tau(r, \Omega, s)} Q(\mathbf{r}-s \boldsymbol{\Omega}, \mathbf{\Omega})
$$

where as before $s_{\mathrm{b}}=s_{\mathrm{b}}(\mathbf{r}, \boldsymbol{\Omega})$ is the path length to the boundary of the domain (either the surface of the nucleus or the top of the atmosphere), $Q(\mathbf{r}, \mathbf{\Omega})$ is a known source term, and the optical depth was defined in Eq. (15).

\section{Evaluation of Optical Depths}

We assume the functional form of the extinction is known. For the case of constant radial outflow from a point source (spherically symmetric coma) all optical depths can be found analytically:

$$
\begin{aligned}
& \tau(\mathbf{r}, \mathbf{\Omega}, s)=R \tau_{0} \int_{0}^{s} \frac{d s^{\prime}}{\mid \mathbf{r}-s^{\prime} \mathbf{\Omega} !^{2}} \\
& \quad=\left\{\begin{array}{ll}
\frac{R \tau_{0}}{\sqrt{r^{2}-(\mathbf{r} \cdot \mathbf{\Omega})^{2}}}\left\{\tan ^{-1}\left(\frac{s-\mathbf{r} \cdot \mathbf{\Omega}}{\sqrt{r^{2}-(\mathbf{r} \cdot \mathbf{\Omega})^{2}}}\right)\right. \\
\left.-\tan ^{-1}\left(\frac{-\mathbf{r} \cdot \mathbf{\Omega}}{\sqrt{r^{2}-(\mathbf{r} \cdot \mathbf{\Omega})^{2}}}\right)\right\} & \text { for }|\mathbf{r} \cdot \mathbf{\Omega}|<r \\
\frac{R \tau_{0} s}{r(r+s)} & \text { for } \mathbf{r} \cdot \mathbf{\Omega}=-r \\
\frac{R \tau_{0} s}{r(r-s)} & \text { for } \mathbf{r} \cdot \mathbf{\Omega}=r
\end{array}\right\} .
\end{aligned}
$$

For the general case, in particular the axisymmetric jet, integration is performed by Romberg integration using library software.

\section{Evaluation of Path Integrals for Solution to $I_{1}$.}

For this case, the source function, $Q$, in Eq. (Al) is known throughout the domain and is given by

$$
Q(\mathbf{r}, \boldsymbol{\Omega})=\frac{S \lambda}{4} \alpha(\mathbf{r}) P\left(\boldsymbol{\Omega} \cdot \mathbf{\Omega}_{\mathrm{o}}\right) e^{-r\left(\mathrm{r}, \boldsymbol{\Omega}_{\mathrm{v}}, S_{\mathrm{b}}\left(\mathbf{r}, \Omega_{\mathrm{o}}\right)\right.}
$$

Evaluation of the integral in Eq. (A1) is performed by Romberg integration using library software.

\section{Evaluation of Path Integrals for Lambda Iteration Solution for $I_{z 2}$.}

The lambda iteration step involves evaluating an integral of the form of Eq. (A1) for each position point $\left(r_{j}, \theta_{i}\right)$ and for each discrete direction

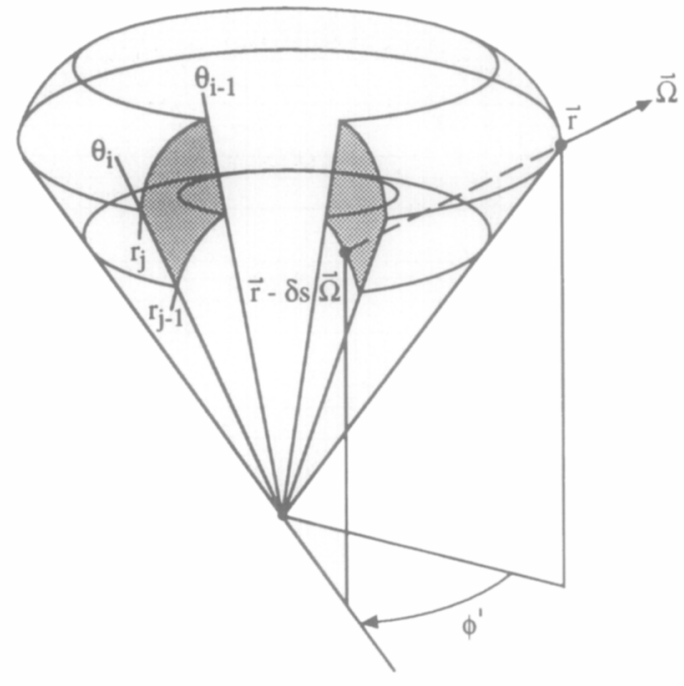

FIG. 10. The spatial domain is discretized in $r$ and $\theta$. For numerical integration purposes, an increment of domain appears as a spherical annulus. Each step in the integration scheme traverses an incremental spherical annulus.

ordinate in the atmosphere. The spatial dependence of the source term $Q$ is known only at discrete positions in the domain, but it is supposed that the direction dependence of $Q$ is known for arbitrary $\Omega$. The position domain is discretized in $r$ and $\theta$ but is formally considered continuous in the suppressed variable $\phi$. Therefore, in the discretization scheme, each "increment of domain," defined by $\left[r_{j-1}, r_{j}\right]$ and $\left[\theta_{i-1}, \theta_{i}\right]$, is a spherical annulus enclosed by two neighboring radial surfaces and two neighboring angular surfaces. A sample "increment of domain" is shown in Fig. 10.

To illustrate the scheme, we begin the path integration at a grid point $\left(r_{j}, \theta_{i}\right)$ and consider a direction ordinate $\Omega$. The grid point lies on an edge of the spherical annulus described above. Proceeding along a path in the fixed direction $-\Omega$, the code determines the path length $\delta s$ such that $\mathbf{r}-\delta s \Omega$ is the point where the path exists the annulus (see Fig. 10).

The exit point lies on a surface of either constant $r$ or constant $\theta$. Due to rotational symmetry previously discussed, azimuthal spatial dependence never explicitly appears in the solution, so the lines of discretization can be represented by a grid in the $r-\theta$ plane. The direction $\Omega$ is constant, but the projection of the path in the $r-\theta$ plane appears curved.

In general the coordinates of the starting point will be $(r, \theta, \phi=0)$, and the coordinates of the exit point will be $\left(r^{\prime}, \theta^{\prime}, \phi^{\prime}\right)$, where the spatial azimuth $\phi^{\prime}$ at the exit is in general not zero. To utilize the symmetry of the problem we transform the direction at the exit to the "local" reference: $\mathbf{\Omega}^{\prime}=\mathbf{R}_{i}\left(\phi^{\prime}\right) \cdot \mathbf{\Omega}$.

The value of the source function at the exit point on the annulus, $Q_{1}$, is determined by linear interpolation. If the exit point is on a constant$r$ surface $\left(r^{\prime}=r_{j-1}\right.$, for example, as in Fig. 10), we interpolate between angular grid points,

$$
\begin{aligned}
Q_{1}=\frac{1}{\theta_{i}-\theta_{i-1}}\left(\left(Q\left(r_{j-1}, \theta_{1}, \mathbf{\Omega}^{\prime}\right)-\right.\right. & \left.Q\left(r_{j-1}, \theta_{i-1}, \mathbf{\Omega}^{\prime}\right)\right)\left(\theta-\theta_{i-1}\right) \\
& \left.+\left(\theta_{i}-\theta_{i-1}\right) Q\left(r_{j-1}, \theta_{i-1}, \mathbf{\Omega}^{\prime}\right)\right)
\end{aligned}
$$

In a similar manner we perform linear interpolation between radial grid points if the exit point is on a constant $-\theta$ surface. Next it is assumed that $Q$ varies linearly along the path from the initial point to the point of exit 


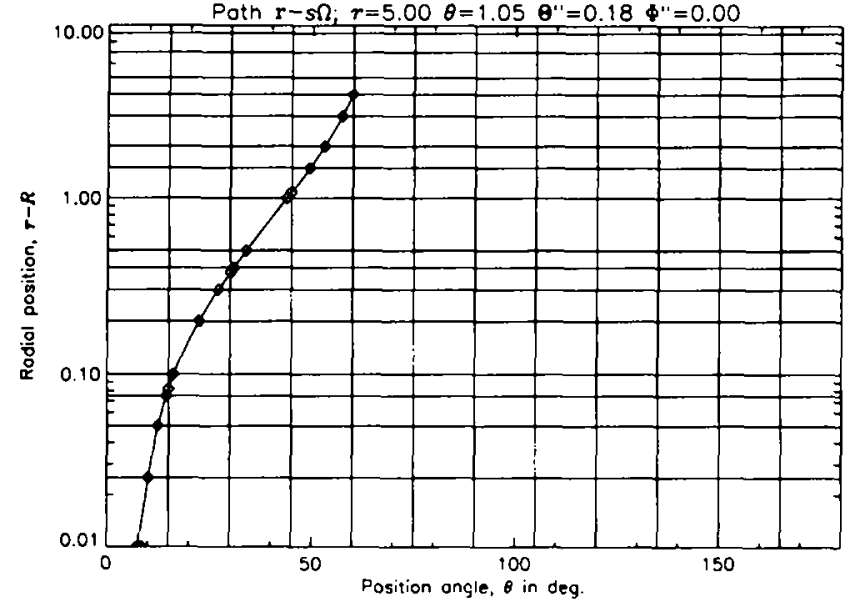

FIG. 11. The path of integration appears curved in the $r-\theta$ plane. This particular integration path intersects the nucleus. The code determines the $r-\theta$ mesh crossing points, shown as diamonds. An independent calculation of the continuous path is superposed for reference (but never used in the numerical integration scheme).

from the annulus, so trapezoidal rule integration is performed using the initial value, $Q_{0}$, the interpolated value at the exit, $Q_{1}$, and the determined path length from initial point to exit of annulus, $\delta s$.

$$
\delta I_{1}=\frac{1}{2}\left(Q_{1} e^{-;(r, \Omega . \delta s)}+Q_{0}\right) \delta s .
$$

Finally, we update the position, $(r, \theta) \rightarrow\left(r^{\prime}, \theta^{\prime}\right)$, and the local direction, $(\Theta, \Phi) \rightarrow \mathbf{R}_{i}\left(\phi^{\prime}\right) \cdot(\Theta, \Phi)$. This procedure is then repeated across each increment of domain, until the path either intersects the nucleus or exits the top of the atmosphere. In general we have

$$
\delta I_{k}=\frac{1}{2}\left(Q_{k} e^{-\gamma\left(\mathrm{r}, \boldsymbol{\Omega}, s_{k}+\delta s_{k}\right)}+Q_{k-1} e^{-\tau\left(\mathrm{r}, \boldsymbol{\Omega} . s_{k}\right)}\right) \delta s_{k}
$$

where $s_{k}$ is the path length from the initial grid point $r_{j}$ to the starting point of integration at the $k$ th step, and $\delta s_{k}$ is the path length across the incremental spherical annulus at that step. Including the boundary condition, the total path integral is

$$
I(\mathbf{r}, \mathbf{\Omega}) \cong I\left(\mathbf{r}-s_{b} \mathbf{\Omega}, \mathbf{\Omega}\right) e^{\left.-\boldsymbol{r ( r , \Omega ,}, s_{\mathrm{h}}(\mathbf{r}, \Omega)\right)}+\sum_{k} \delta I_{k}
$$

Figure 11 shows a complete path of integration, and the code's independent evaluation of the mesh crossing points is superposed. This particular path starts at 5 radii from the origin and eventually intersects the nucleus surface.

\section{APPENDIX 2. SPECIFICATION OF DIRECTION ORDINATES}

At each grid point in the position domain, we solve the radiative transfer equation in discrete direction ordinates. In order to distribute the ordinates efficiently over the domain, and in a way that will permit quadrature consistent with the limb discontinuity in the problem, we define a position-dependent division of the direction domain. Ordinates are defined relative to the local spherical reference. We denote angles in this reference by a double prime.
For a given position, we divide the local direction angle domain into three regions (refer to Fig. 12): Region one is zenith-pointing directions corresponding to paths of integrations which intersect the nucleus. The nucleus' limb appears at angle $\beta(r)=\sin ^{-1}(R / r)$ in the local spherical $\boldsymbol{\Omega}^{\prime \prime}$ system. Region two is directions corresponding to integration paths which pass within a somewhat arbitrarily chosen angle, $\gamma(r)$, off the nucleus. Region three is the remaining direction domain, for which paths of integration extend zenithward toward open space.

The angle $\gamma(r)$ is chosen in the following way. We first specify its values at $r_{\max }$ and at $R$. For positions far from the nucleus, we need $\gamma$ such that integration paths are concentrated just off the limb of the nucleus. We will arbitrarily set $\gamma\left(r_{\max }\right)=\sin ^{-1}\left(3 R / r_{\max }\right)$, corresponding to an integration path passing 2 comet radii off the limb. On the other hand, in the sunward half of the coma near the nucleus surface we need $\gamma$ such that integration paths extend near the Sun. For this condition we distribute the ordinates uniformly over the open sky. So, again somewhat arbitrarily, we set $\gamma(R)=\cos ^{-1}(-1 / 2)$. Finally the radial dependence of $\gamma$ is set so that $\gamma(r)$ varies linearly between the near-nucleus and the far-nucleus limits,

$$
\begin{aligned}
\cos \gamma(r)=\left[\left(\cos \gamma\left(r_{\max }\right)+1 / 2\right)(r-R)\right. & \\
& \left.-\left(r_{\max }-R\right) / 2\right] /\left(r_{\max }-R\right) .
\end{aligned}
$$

Integration over each angular region is performed with GaussLegendre quadrature. The azimuth integral is treated with uniformly spaced trapezoidal rule quadrature of order $M$. In quadrature form the scattered-light source term for a fixed direction $\boldsymbol{\Omega}_{q}$ is then

$$
\begin{aligned}
& \oint_{4 \pi} d \boldsymbol{\Omega} P\left(\boldsymbol{\Omega} \cdot \boldsymbol{\Omega}_{q}\right) /(\boldsymbol{\Omega}) \\
& \begin{array}{c}
\cong \frac{2 \pi}{M-1} \sum_{m=1}^{M}\left\{\sum_{i=1}^{N_{3}} \frac{1}{2}(\cos \gamma+1) w_{i}^{\prime} P\left(\left(\mathbf{\Omega}^{\prime \prime} \cdot \mathbf{\Omega}_{q}^{\prime \prime}\right)_{i, m}\right)\right. \\
\times I\left(\mathbf{R}_{z}(-\phi) \cdot \mathbf{R}_{y}(-\theta) \cdot \mathbf{\Omega}_{i, m}^{\prime \prime}\right)
\end{array} \\
& +\sum_{j=1}^{v_{2}} \frac{1}{2}(\cos \beta-\cos \gamma) w_{j} P\left(\left(\boldsymbol{\Omega}^{\prime \prime} \cdot \boldsymbol{\Omega}_{q}^{\prime \prime}\right)_{j, m}\right) \\
& \times I\left(\mathbf{R}_{i}(-\phi) \cdot \mathbf{R}_{y}(-\theta) \cdot \mathbf{\Omega}_{j, m}^{\prime \prime}\right) \\
& +\sum_{k=1}^{N_{1}} \frac{1}{2}(1-\cos \beta) \boldsymbol{w}_{k} P\left(\left(\mathbf{\Omega}^{\prime \prime} \cdot \mathbf{\Omega}_{q}^{\prime \prime}\right)_{k, m}\right) \\
& \left.\times I\left(\mathbf{R}_{i}(-\phi) \cdot \mathbf{R}_{y}(-\theta) \cdot \mathbf{\Omega}_{k, m}^{\prime \prime}\right)\right\},
\end{aligned}
$$

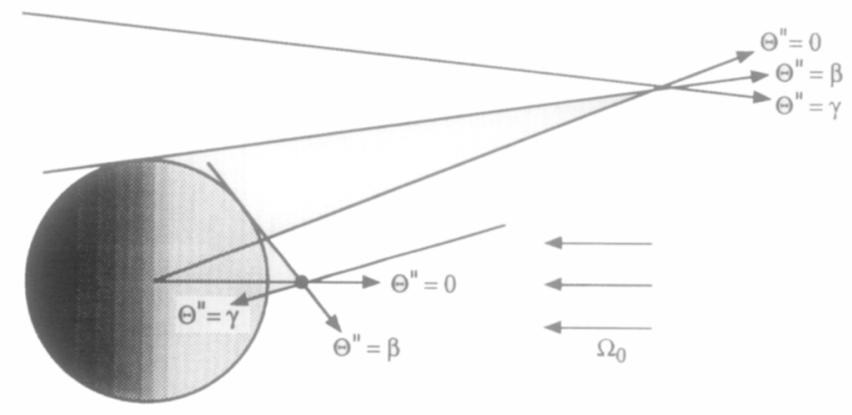

FIG. 12. The angle parameters $\beta$ and $\gamma$ used to define discrete direction angles $\Theta$ ". For positions distant from the nucleus, $\gamma$ is set so integration paths pass near the limb. For positions near the nucleus, $\gamma$ is set to distribute ordinates fairly uniformly over the open sky. 
where $N_{1}, N_{2}$, and $N_{3}$ are Gauss-Legendre quadrature orders, and the $w$ are weights for the interval $[-1,1]$. Subscripts on the directiondependent quantities specify the direction abscissa in which the quantity is to be evaluated; for example, $\boldsymbol{\Omega}_{l, m}^{\prime \prime}$ is to be evaluated with $\Theta$ " = $\cos ^{-1}\left(\zeta_{i}\right)$ where $\zeta_{i}$ is the $i$ th abscissa for Gauss-Legendre quadrature of order $N_{3}$, scaled to the interval [ $-1, \cos (\gamma)$ ], and with $\Phi^{\prime \prime}=\pi m /(M-$ 1). Therefore, once $\gamma(r), M, N_{1}, N_{1}$, and $N_{3}$ are chosen, and $\beta(r)$, is calculated, the quadrature points and weights are determined. For the work presented here we have used $N_{1}=2, N_{2}=3, N_{3}=2$ to 5 , and $M=5$ or 7 .

\section{APPENDIX 3. NOMENCLATURE}

\section{Scalars}

$g \quad$ Henyey-Greenstein scattering parameter

$m \quad$ mass per particle

$n \quad$ number density

$r \quad$ radial position

$r_{\max } \quad$ maximum radial distance

$s \quad$ path length

$s_{b} \quad$ path length to boundary of domain

$v \quad$ radial speed

$\boldsymbol{w}_{i} \quad$ Gauss-Legendre quadrature weight

$F_{\text {vis }} \quad$ total flux of visible light

$F_{\text {vis(dir) }}$

$F_{\text {vis(ms) }}$

$F_{\text {vis(ss) }}$

flux of visible direct (unscattered) light

flux of visible multiple scattered light

flux of visible single scattered light

$\bar{F}_{\text {vis(ss) }}$

flux of visible single scattered light, averaged over the nucleus surface

$F_{\pi} \quad$ flux of reradiated energy

$F_{\text {midir) }} \quad$ direct component of flux of reradiated energy

$F_{\text {m(ms) }} \quad$ multiple scattered component of flux of reradiated energy

I specific intensity

$I_{0} \quad$ specific intensity of unscattered light

$I_{1} \quad$ specific intensity of single-scattered light

$I_{\geq 2} \quad$ specific intensity of light scattered two or more times

$I_{\geq 2}^{k} \quad$ specific intensity of light scattered two or more times on the $k$ th iteration step

$J_{\text {vis(ms) }} \quad$ flux of visible multiply scattered light

$M \quad$ trapezoidal rule quadrature order

$N_{1}, N_{2}, N_{3}$ Gauss-Legendre quadrature orders

$P \quad$ phase function

$q \quad$ source strength

$Q \quad$ source function (in Appendix 1)

$Q_{k} \quad$ source function at the $k$ th point in trapezoidal rule integration

$R \quad$ radius of model comet

$S \quad$ solar constant

$X \quad$ jet strength

$\alpha \quad$ extinction

$\beta \quad$ direction angle parameter to define discrete ordinates

$\delta_{2} \quad$ two-dimensional delta function

$\delta s \quad$ increment of path length

$\delta s_{k} \quad$ increment of path length at $k$ th step in trapezoidal rule integration

$\delta I \quad$ increment of specific intensity

$\delta I_{k} \quad$ increment of specific intensity at $k$ th step in trapezoidal rule integration

ith Gauss-Legendre quadrature point

direction angle parameter to define discrete ordinates

single scatter albedo

mass density

total cross section for scattering optical depth

optical depth of a spherically symmetric coma

position angle

jet angular width

position azimuth

direction angle

direction angle in spherical reference

direction azimuth

direction azimuth in spherical reference

\section{Vectors and Matrices}

\section{$\mathbf{e}_{\mathrm{x}}, \mathbf{e}_{\mathrm{y}}, \mathbf{e}_{\mathrm{z}} \quad$ unit vectors along Cartesian axes}

$\mathbf{r}$

$\mathbf{R}_{i}(\Phi) \quad$ rotation about the $z$-axis through angle $\phi$

$\mathbf{\Omega} \quad$ direction

$\mathbf{\Omega}^{\prime} \quad$ incident direction

$\boldsymbol{\Omega}_{\mathrm{o}} \quad$ direction of incident solar rays

$\boldsymbol{\Omega}_{q} \quad$ an arbitrary fixed direction

$\boldsymbol{\Omega}^{\prime \prime} \quad$ direction in the spherical reference.

\section{ACKNOWLEDGMENTS}

This work was supported by NSF Grant AST-9015928 and by NASA Grant NAGW 1366. Acknowledgments are also made to the University of Michigan and to the National Center for Atmospheric Research sponsored by the NSF for the computing time used in this research.

\section{REFERENCES}

CASE, K. M., AND P. F. Zweifel. 1967. Linear Transport Theory. Addison-Wesley, Reading, MA.

Case, K. M., F. DeHoffmanN, and G. Placzex 1953. Introduction to the Theory of Neutron Diffusion. Los Alamos Scientific Laboratory, Los Alamos, NM.

Cheyney, H., ANd A. ARking 1976. A new formulation for anisotropic radiative transfer problems. I. Solution with a variational technique. Astrophys. J. 207, 808-819.

Festou, M. C., G. P. Tozzi, L. A. Smaldone, P. Felenbor, R. FAl.CIANi, AND J. M. ZuCCONI 1990. Did an outburst occur on 4 December 1985 in Halley's comet? Astron. Astrophys. 227, 609-618.

Germogenova, T. A., L. I. Koprova, and T. A. Sushrevich 1969. Investigation of the angular distribution and spectral structure of the terrestrial radiation field for characteristic model spherical atmospheres. Izv. Acad. Sci. USSR, Atmos. Oceanic Phys. 5, 731-737.

Gombosi, T. I., A. F. NagY, and T. E. Cravens 1986. Dust and neutral gas modeling of the inner atmospheres of comets. Rev. Geophys. 24, $667-700$.

HellMiCH, R. 1981. The influence of the radiation transfer in cometary dust halos on the production rates of gas and dust. Astron. Astrophys. 93, 341-346.

Herman, G., and H. Salo 1987. Light scattering in cometary dust comae. Earth Moon Planets 89, 51-74.

Huebner, W. F., D. C. Boice, H. J. Reitsema, W. A. Delamere, AND F. L. WhIPPLE 1988. A model for intensity profiles of dust jets near the nucleus of Comet Halley. Icarus 76, 78-88.

Hughes, D. W. 1991. Possible mechanisms for cometary outbursts. In Comets in the Post-Halley Era (R. L. Newburn, Jr., M. Neugebauer, and J. Rahe, Eds.), pp. 825-851. Kluwer, Dordrecht.

JewItT, D. 1991. Cometary photometry. In Comets in the Post-Halley 
Era (R. L. Newburn, Jr., M. Neugebauer, and J. Rahe, Eds.), Vol. 1. pp. 19-65. Kluwer, Dordrecht.

Keller, H. U., M. L. Marconi, and N. Thomas 1990. Hydrodynamic implications of particle fragmentation near cometary nuclei. Astron. Astrophys. 227, L1-L4.

KelleR, H. U., ANd N. Thomas 1989. Evidence for near-surface breezes on comet P/Halley. Astron. Astrophys. 226, L9-L12.

Keller, H. U., W. A. Delamere, W. F. Huebner, H. J. Reitsema, H. L. Schmidt, F. L. Whipple, K. Wilhel.M, W. Curdt, R. Kramm, N. Thomas, C. Arpigny. C. Barbieri, R. M. Bonnet, S. Cazes, M. Coradini, C. B. Cosmovici, D. W. Hughes, C. Jamar, D. Malaise, K. Schmidt, W. K. H. Schmidt, and P. Seige 1987. Comet P/Halley's nucleus and its activity. Astron. Astrophys. 187, 807-823.

Kitamura, Y. 1986. Axisymmetric dusty gas jet in the inner coma of a comet. Icarus 66, 241-257.

Köml.e, N. I., G. Dettleff, And C. Dankert 1990. Thermal behaviour of pure and dusty ices on comets and icy satellites. Astron. Astrophys. 227, 246-254.

LARSEN, E. W. 1988. Solution of three dimensional inverse transport problems. Transp. Theory Stat. Phys. 17, 147-167.

Larson, H. P., H.-Y. Hu, M. J. Mumma, and H. A. Weaver 1990. Outbursts of $\mathrm{H}_{2} \mathrm{O}$ in Comet $\mathrm{P} /$ Halley. Icarus 86, 129-151.

Lamy, P. L., P. Malburet, A. Llebaria, and S. Koutchmy 1989. Comet P/Halley at a heliocentric preperihelion destance of $2.6 \mathrm{AL}$ : Jet activity and properties of the dust coma. Astron. Astrophys. 222, 316-322.

LENOBI.F. J., AND Z. SEKERA 1961. Equation of radiative transfer in a planetary spherical atmosphere. Proc. Natl. Acad. Sci. USA 47, 372-378.

Marconi, M. L., ANd D. A. Mendis 1984. The effects of the diffuse radiation fields due to multiple scattering and thermal reradiation by dust on the dynamics and thermodynamics of a dusty cometary atmosphere. Astrophys. J. 287, 445-454.

Marconi, M. L., AND D. A. MEndis 1986. Infrared heating of comet Halley's atmosphere. Earth Moon Planets 36, 249-256.

METZ, K., AND R. HAFF.NER 1987. Circular polarization near the nucleus of Comet P/Halley. Astron. Astrophys. 187, 539-542.
MuKaI, S., T. MUKaI, AND S. KIKUChI 1985. Intensity profiles of the multiple scattering light near the cometary nucleus. Adv. Space Res. 5, 89-92.

Ney, E. P., AND Merril. , K. M. 1976. Comet West and the scattering function of cometary dust. Science 194, 1051-1053.

Reitsema, H. J., W. A. Delamere, A. R. Williams, D. C. Boice, W. F. HeUBNer, AND F. L. Whipple 1989a. Dust distribution in the inner coma of Comet Halley: Comparison with models. Icarus 81, $31-40$.

Reitsema, H. J., W. A. Delamere, and F. L. Whipple 1989b. Active polar region on the nucleus of comet Halley. Science 243, 198-200.

SALo, H. 1988. Monte Carlo modeling of the net effects of coma scattering, and thermal reradiation on the energy input to cometary nucleus. Icarus 76, 253-269.

Sekanina, Z. 1987. Anisotropic emission from comets: Fans versus jets. I. Concepts and modeling. In Symposium on the Diversity and Similarity of Comets, pp. 315-322. European Space Agency, Noordwijk, the Netherlands.

Sen, A. K., U. C. Joshi, M. R. Deshrande, and C. Debi Prasad 1990. Imaging polarimetry of Comet P/Halley. Icarus 86, 248-256.

SHIA, R.-L., AND Y. L. YUNG 1986. Radiative transfer in a sphere illuminated by a parallel beam: An integral equation approach. Astrophys. J. 301, 554-570.

Sobol.fv, V. V. 1975. Light Scattering in Planetary Atmospheres. Pergamon, New York.

SQuyres, S. W., C. P. MCKey, and R. T. Reynolds 1985. Temperatures within comet nuclei. J. Geophys. Res. 90, 12381-12392.

ThOMAS, N., AND H. L. KelleR 1990. Interpretation of the inner coma observations of comet $\mathrm{P} / \mathrm{Halley}$ by the Halley multicolour camera. Ann. Geophys. 8, 147-160.

Thomas, N., D. C. Boice, W. F. Huebner, and H. U. Keller 1988. Intensity profiles of dust near extended sources on comet Halley. Nature 332, 51-52.

Weissman. P. R., AND H. H. KiefFer 1981. Thermal modeling of cometary nuclei. Icarus 47, 302-311.

WII.SON. S. J., AND K. K. SEN 1980. Light scattering by an optically thin inhomogeneous spherically symmetric planetary atmosphere. Astrophys. Space Sci. 69, 107-113. 